\title{
Measurement and Correlation of Liquid-Liquid Equilibrium Data of Mesityl oxide-Diethoxymethane-Water System
}

\author{
Hongyue Guo ${ }^{1}$, Mai Han ${ }^{1}$, Yicong Zhao ${ }^{1}$ and Qingsong Li $^{1 *}$ \\ ${ }^{1}$ The State Key Lab of Heavy Oil Processing, College of Chemical Engineering, China University of Petroleum (East China), Huangdao, \\ Qingdao, Shandong, China;
}

\begin{abstract}
To provide essential data for the separation of diethoxymethane and system water using mesityl oxide as the extractant, in this paper, the liquid-liquid equilibrium data of the ternary system of 'mesityl oxide + diethoxymethane + water' was measured at $303.2 \mathrm{~K}, 313.2 \mathrm{~K}$ and $323 \mathrm{~K}$ under normal pressure. The experimental results showed that partition coefficient and separation factor were both larger than 1 , indicating that diethoxymethane and water could be well separated with mesityl oxide. The linear correlation coefficient of Bachman and Hand equation was larger than 0.99 , indicating that our experimental data has good reliability. At the same time, the binary interaction parameters of the model were obtained by correlating the experimental data with the NRTL and UNIQUAC models. The relative root mean square error (RMSD) of the experimental value and the calculation formula was less than $0.79 \%$, indicating that both the NRTL and UNIQUAC models can be well associated with experimental data.
\end{abstract}

\section{Introduction}

Diethoxymethane is an important organic substance, which is widely used in chemical industry, pharmaceutical separation, synthesis of organic intermediates and reaction solvents [1]. In addition, diethoxymethane is also an excellent diesel fuel additive, which can increase the octane number and reduce carbon monoxide emissions [2]. At present, diethoxymethane is mainly synthesized by formaldehyde and ethanol, however, diethoxymethane is easy to form azeotropes with by-products of water and ethanol during the synthesis process [3], which makes it difficult to obtain high-purity diethoxymethane and cause water pollution. Solvent extraction method can well overcome this azeotropic effect. After screening, it was found that mesityl oxide was an excellent extractant [4], which is usually used as a solvent for nitrocellulose and various resins.

As an effective separation method, solvent extraction has been widely used in various industries, including wastewater treatment [5-8], pharmaceuticals [8-10] and food engineering [11,12]. Reliable liquid-liquid balance data plays an important role in the extraction and separation process and equipment design. Therefore, many researchers have studied the liquid-liquid equilibrium $[13,14]$. The research group Wang [15] et al. studied the liquid-liquid equilibrium of the 'diethoxymethane + water + toluene/ p-xylene' ternary system. In this paper, the liquid-liquid equilibrium data of the ternary system of 'mesityl oxide + diethoxymethane + water' at $303.2 \mathrm{~K}, 313.2 \mathrm{~K}$ and $323.2 \mathrm{~K}$ were measured under normal pressure. By consulting the literature, no research about the mesityl oxide-diethoxymethane-water ternary system has been published. At the same time, the extraction effect of mesityl oxide was evaluated by calculating the partition coefficient and selectivity coefficient, and the thermodynamic consistency of the experimental data was checked by the equations of Bachman [16] and Hand [17]. Finally, NRTL [18] and UNIQUAC [19] model was associated with experimental data.

\section{Experimental process and method}

\subsection{Apparatus and reagents}

The reagents used in the experiment are shown in Table 1. Their purities were determined by chromatographic analysis. All the reagents were used directly without further purification treatment; the phase equilibration kettle is shown in Figure 1.

\subsection{Experimental process and analysis method}

In our experiment, an electronic analytical balance was used to weigh the quantitative components, and then they were added to the liquid-liquid equilibrium kettle

"Corresponding author E-mail: licup01@163.com 
Table 1 Chemical reagents ${ }^{\mathrm{a}}$

\begin{tabular}{|c|c|c|c|c|}
\hline Compound & CAS & Supplier & $\begin{array}{l}w \\
/ \%\end{array}$ & $\begin{array}{c}\text { Purity } \\
\text { analysis } \\
\text { method }\end{array}$ \\
\hline Mesityl oxide & $\begin{array}{l}141- \\
79-7\end{array}$ & Aladdin & $\begin{array}{c}> \\
99.0\end{array}$ & $\mathrm{GC}^{\mathrm{b}}$ \\
\hline Diethoxymethane & $\begin{array}{l}462- \\
95-3\end{array}$ & Aladdin & $\begin{array}{c}> \\
99.5\end{array}$ & $\mathrm{GC}^{\mathrm{b}}$ \\
\hline Distilled water & $\begin{array}{c}7732- \\
18-5\end{array}$ & Homemade & $\begin{array}{c}> \\
99.9\end{array}$ & $\mathrm{GC}^{\mathrm{b}}$ \\
\hline
\end{tabular}

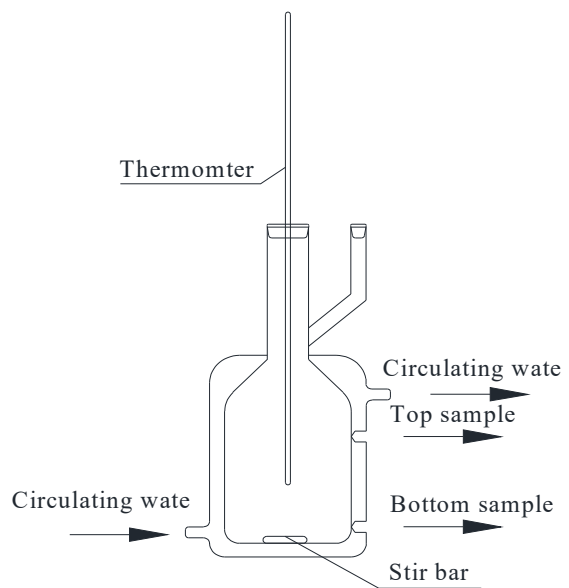

Figure 1 Phase Equilibration kettle

in different proportions. And then, the thermostatic jacket was connected with the super thermostatic water bath to keep the temperature in the equilibrium kettle constant, stir for 2 hours, and let it stand for 8 hours after the stirring stops to ensure that the two phases were fully balanced. After equilibration, samples were taken from the upper and lower sampling ports of the balance tank by using a micro-injector for analysis. The internal standard method was used with gas chromatography (TCD) to quantitatively analyze the upper and lower samples to determine the mass fraction of each component, and isopropanol was used as the internal standard. Each sample was measured at least 3 times to ensure that the standard deviation is less than $0.1 \%$, and the average value was used as the experimental value, and the uncertainty of the experimental value was calculated by using the GUM [20] standard.
Chromatographic analysis conditions: the chromatographic column of TCD (Agilent GC6820) was Porapak N ( $3 \mathrm{~mm} \times 3 \mathrm{~m}$ ) ; high-purity hydrogen was used as the carrier gas, and the gas velocity was $60 \mathrm{~mL} / \mathrm{min}$; the temperature of the vaporization chamber and the detector were both $493.15 \mathrm{~K}$, the initial column temperature was maintained at $403.15 \mathrm{~K}$ for $0.5 \mathrm{~min}$, and the temperature was programmed at $15 \mathrm{~K} / \mathrm{min}$ to $473.15 \mathrm{~K}$ and maintained for $1 \mathrm{~min}$. The injection volume was 0.6 $\mathrm{uL}$.

\section{Experimental results and discussion}

\subsection{Experimental data}

The liquid-liquid equilibrium data is shown in Table 2, and all the compositions are expressed in mass fraction. The ternary phase diagram is shown in Figure 2. From the ternary phase diagram, it can be seen that the two-phase region has a larger range for extraction operations. The partition coefficient and selectivity coefficient can evaluate the extraction ability of the extractant [5]. The formulas of distribution coefficient and selectivity coefficient are as follows:

$$
\begin{aligned}
& D=w_{2}^{\mathrm{I}} / w_{2}^{\mathrm{II}} \\
& S=\left(w_{2}^{\mathrm{I}} \cdot w_{3}^{\mathrm{II}}\right) /\left(w_{2}^{\mathrm{II}} \cdot w_{3}^{\mathrm{I}}\right)
\end{aligned}
$$

Among them, $w_{2}^{\mathrm{I}}$ and $w_{3}^{\mathrm{I}}$ represent the mass fraction of diethoxymethane and water in the organic phase respectively, and $w_{2}^{\mathrm{II}}$ and $w_{3}^{\mathrm{II}}$ represent the mass fraction of diethoxymethane and water in the water phase, respectively. The partition coefficient and selectivity coefficient are shown in Table 2, and the partition coefficient and selectivity coefficient are shown in Figure 3 and Figure 4. Through analysis, it can be known that mesityl oxide has a good extraction effect on diethoxymethane. The partition coefficient increases with the increase of diethoxymethane concentration in the organic phase, and increases with the rise of temperature; at the same time, it can be found that the selectivity coefficient of mesityl oxide is much greater than 1, indicating that mesityl oxide has good selectivity on diethoxymethane and increases with the increase of the diethoxymethane concentration in the organic phase, and at the same time increases with the temperature.

Table 2 The LLE data (mass fraction), distribution coefficient (D) and selectivity coefficient (S) of ternary system of mesityl oxide

\begin{tabular}{|c|c|c|c|c|c|c|c|c|}
\hline \multirow{2}{*}{$\mathrm{T} / \mathrm{K}$} & \multicolumn{3}{|c|}{ Organic (I) } & \multicolumn{3}{|c|}{ Aqueous (II) } & \multirow{2}{*}{$\mathrm{D}$} & \multirow{2}{*}{$\mathrm{S}$} \\
\hline & ${ }^{\mathrm{a}} w^{\mathrm{I}} 1$ & ${ }^{\mathrm{a}} w^{\mathrm{I}}{ }_{2}$ & ${ }^{\mathrm{a}} w^{\mathrm{I}_{3}}$ & $\mathrm{~b}_{w{ }_{1}{ }_{1}}$ & ${ }^{\mathrm{b}} w \mathrm{II}_{2}$ & ${ }^{\mathrm{b}} w \mathrm{II}_{3}$ & & \\
\hline \multirow{7}{*}{303.2} & 0.9658 & - & 0.0355 & 0.0226 & - & 0.9766 & - & - \\
\hline & 0.9296 & 0.0332 & 0.0348 & 0.0215 & 0.0019 & 0.9768 & 17.44 & 489.52 \\
\hline & 0.8725 & 0.0930 & 0.0345 & 0.0189 & 0.0042 & 0.9775 & 21.96 & 622.22 \\
\hline & 0.8440 & 0.1204 & 0.0339 & 0.0176 & 0.0052 & 0.9778 & 23.33 & 673.99 \\
\hline & 0.8127 & 0.1524 & 0.0335 & 0.0153 & 0.0060 & 0.9789 & 25.46 & 744.09 \\
\hline & 0.7780 & 0.1866 & 0.0322 & 0.0147 & 0.0072 & 0.9790 & 25.89 & 786.06 \\
\hline & 0.7386 & 0.2305 & 0.0310 & 0.0137 & 0.0083 & 0.9792 & 27.92 & 883.11 \\
\hline
\end{tabular}
(1) + diethoxymethane (2) + water (3) at 298.2, 303.2 and $313.2 \mathrm{~K}$ under $101.3 \mathrm{kPa}^{*}$. 


\begin{tabular}{rcccccccc}
\hline & 0.6848 & 0.2865 & 0.0289 & 0.0104 & 0.0099 & 0.9798 & 28.94 & 982.23 \\
0.6547 & 0.3240 & 0.0282 & 0.0102 & 0.0107 & 0.9807 & 30.18 & 1048.59 \\
0.6169 & 0.3573 & 0.0281 & 0.0095 & 0.0111 & 0.9811 & 32.05 & 1120.85 \\
0.9612 & - & 0.0388 & 0.0218 & - & 0.9772 & - & - \\
0.9327 & 0.0299 & 0.0374 & 0.0212 & 0.0014 & 0.9775 & 21.88 & 572.46 \\
& 0.8999 & 0.0609 & 0.0367 & 0.0207 & 0.0026 & 0.9775 & 23.42 & 624.47 \\
& 0.8734 & 0.0881 & 0.0374 & 0.0190 & 0.0033 & 0.9777 & 26.90 & 704.05 \\
313.2 & 0.8441 & 0.1185 & 0.0352 & 0.0175 & 0.0043 & 0.9778 & 27.30 & 757.98 \\
& 0.7019 & 0.2676 & 0.0316 & 0.0128 & 0.0089 & 0.9794 & 29.94 & 928.98 \\
& 0.7048 & 0.2720 & 0.0305 & 0.0123 & 0.0090 & 0.9786 & 30.18 & 968.00 \\
& 0.6472 & 0.3212 & 0.0300 & 0.0118 & 0.0099 & 0.9796 & 32.38 & 1057.56 \\
& 0.6139 & 0.3529 & 0.0297 & 0.0107 & 0.0104 & 0.9795 & 33.79 & 1114.13 \\
& 0.5897 & 0.3803 & 0.0291 & 0.0103 & 0.0107 & 0.9799 & 35.68 & 1202.62 \\
0.9617 & - & 0.0383 & 0.0186 & - & 0.9806 & - & - \\
0.9318 & 0.0305 & 0.0377 & 0.0184 & 0.0010 & 0.9806 & 30.33 & 789.75 \\
& 0.9133 & 0.0500 & 0.0372 & 0.0180 & 0.0016 & 0.9807 & 31.05 & 818.46 \\
0.8764 & 0.0889 & 0.0363 & 0.0172 & 0.0028 & 0.9810 & 31.56 & 853.26 \\
0.8486 & 0.1166 & 0.0348 & 0.0162 & 0.0036 & 0.9814 & 32.41 & 914.21 \\
0.8181 & 0.1487 & 0.0338 & 0.0148 & 0.0043 & 0.9816 & 34.64 & 1007.11 \\
0.7877 & 0.1808 & 0.0327 & 0.0133 & 0.0050 & 0.9817 & 36.25 & 1087.27 \\
0.7451 & 0.2208 & 0.0309 & 0.0124 & 0.0060 & 0.9820 & 37.06 & 1179.58 \\
0.7060 & 0.2595 & 0.0301 & 0.0104 & 0.0068 & 0.9824 & 38.15 & 1244.69 \\
0.6134 & 0.3592 & 0.0274 & 0.0092 & 0.0087 & 0.9826 & 41.23 & 1479.15 \\
\hline
\end{tabular}

* Standard uncertainties $\mathrm{u}$ are $\mathrm{u}(\mathrm{T})=0.1 \mathrm{~K}, \mathrm{u}(\mathrm{p})=0.1 \mathrm{kPa}, \mathrm{u}\left(w_{1}\right)=0.002, \mathrm{u}\left(w_{2}\right)=0.0005, \mathrm{u}\left(w_{3}\right)=0.0032$

${ }^{\mathrm{a}} x^{\mathrm{I}}$ : the organic data; ${ }^{\mathrm{b}} x^{\mathrm{II}}$ : the aqueous data.

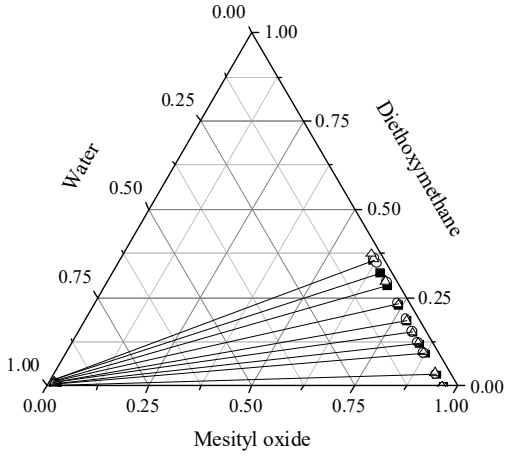

(a)

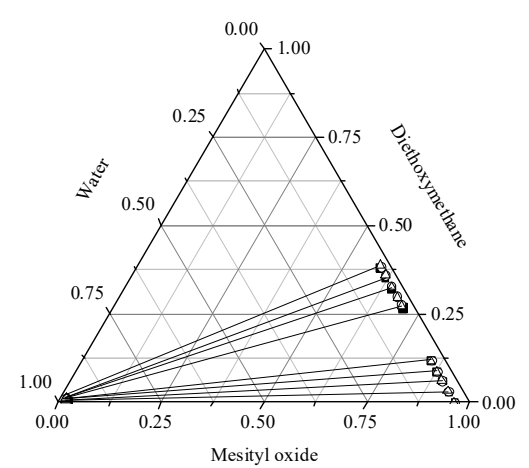

(b)

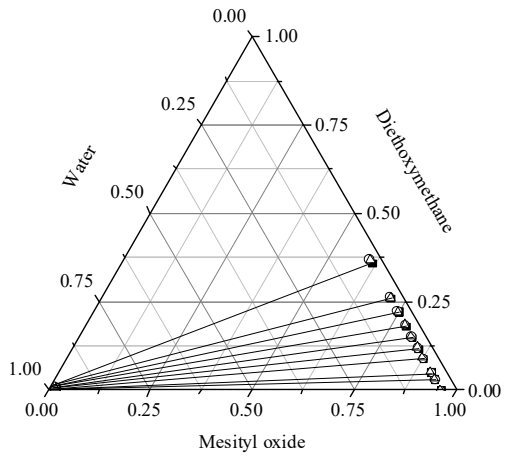

(c)

Figure 2 Ternary diagrams of mesityl oxide (1) + diethoxymethane (2) + water (3) system at different temperatures (a) $303.2 \mathrm{~K}$; (b) $313.2 \mathrm{~K}$; (c) $323.2 \mathrm{~K}$; (

Experimental value; $(\circ)$ NRTL; $\triangle$ ) UNIQUAC;

\subsection{Reliability test of experimental data}

The consistency of the experimental data thermodynamics data was tested by the Bachman [16] and Hand [17] equations, and the formula is as follows:

$$
\begin{gathered}
x_{1}^{\mathrm{I}}=A+B\left(x_{1}^{\mathrm{I}} / x_{3}^{\mathrm{II}}\right) \\
\ln \left(x_{2}^{\mathrm{I}} / x_{1}^{\mathrm{I}}\right)=\mathrm{A}+\mathrm{B} \ln \left(x_{2}^{\mathrm{II}} / x_{1}^{\mathrm{II}}\right)
\end{gathered}
$$

Among them, A, B, C, D represent the Bachman, Hand equation parameters, respectively; $x_{1}^{\mathrm{I}}$ and $x_{2}^{\mathrm{I}}$ represent the mass fraction of mesityl oxide and diethoxymethane in the organic phase respectively; $x_{1}^{\text {II }}, x_{2}^{\text {II }}$ and $x_{3}^{\text {II }}$ represent the mass fraction of mesityl oxide, 
diethoxymethane and water in the water phase respectively.

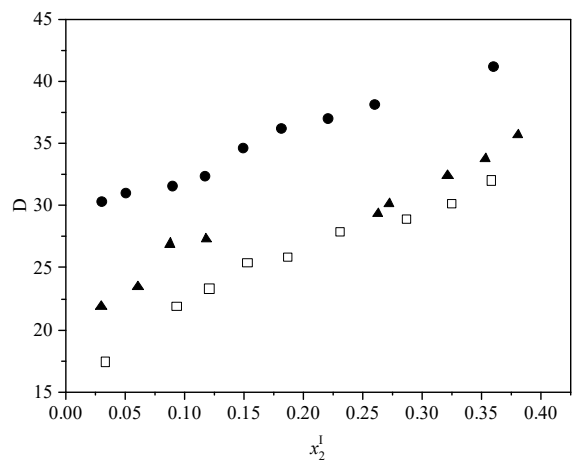

Figure 3 The distribution coefficient versus mass fraction of diethoxymethane in the organic phases 298.2, 303.2, and 313.2 $\mathrm{K}$ under $101.3 \mathrm{kPa}$. (口) $303.2 \mathrm{~K},(\boldsymbol{\Delta}) 313.2 \mathrm{~K}(\bullet) 323.2 \mathrm{~K}$

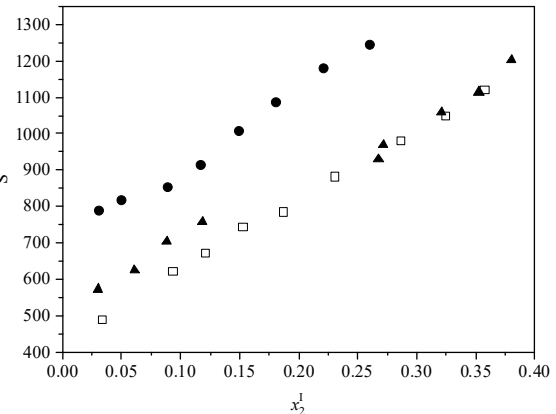

Figure 4. The selectivity coefficient versus mass fraction of diethoxymethane in the organic phases at 298.2,303.2, and $313.2 \mathrm{~K}$ under $101.3 \mathrm{kPa}$. () $303.2 \mathrm{~K},(\boldsymbol{\Delta}) 313.2 \mathrm{~K}(\bullet) 323.2 \mathrm{~K}$

The parameter values and linear correlation coefficients of the Hand equation of the Bachman equation are shown in Table 3. The fitting curves of the Hand equation of the Bachman equation are shown in Figure 5 and Figure 6. Through analysis, it can be seen that the linear coefficients are all close to 1 , indicating that the phase balance data has high consistency and reliability.

Table 3 Bachman and Hand parameters at various temperatures.

\begin{tabular}{ccccccc}
\hline \multicolumn{4}{c}{ Bachman } & \multicolumn{3}{c}{ Hand } \\
\hline $\mathrm{T} / \mathrm{K}$ & $\mathrm{A}$ & $\mathrm{B}$ & $\mathrm{R}$ & $\mathrm{C}$ & $\mathrm{D}$ & $\mathrm{R}$ \\
\hline 303.2 & 0.0080 & 0.9686 & 1.0000 & -2.4213 & -0.98224 & 0.9974 \\
313.2 & 0.0043 & 0.9729 & 1.0000 & -2.5236 & -0.97854 & 0.9992 \\
323.2 & 0.0043 & 0.9762 & 1.0000 & -2.7234 & -1.0310 & 0.9991 \\
\hline
\end{tabular}

\subsection{Thermodynamic model}

This paper uses Aspen Plus software, and uses NRTL [18] and UNIQUAC [19] thermodynamic models to correlate laboratory data. The surface area (r) and van der Waals surface parameters (q) of the UNIQUAC model were obtained from the Aspen Plus database, as shown in Table 4

The least square method is used to correlate the experimental data to minimize the objective function $(O F)$ [21], so as to obtain the best parameters of the

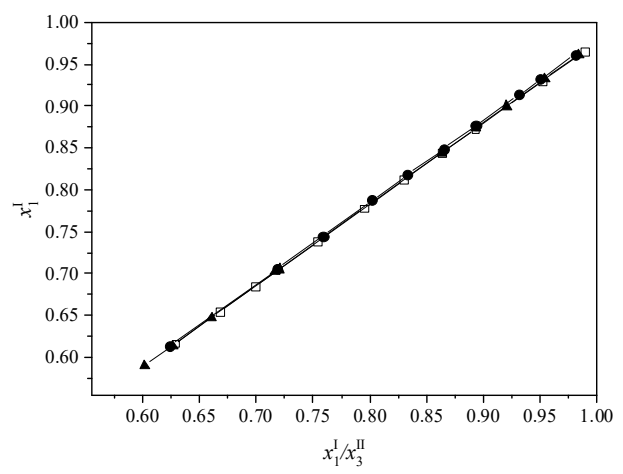

Figure 5 Bachman plots for diethoxymethane at different temperatures. (口) $303.2 \mathrm{~K},(\boldsymbol{\Delta}) 313.2 \mathrm{~K}$ (•) $323.2 \mathrm{~K}$

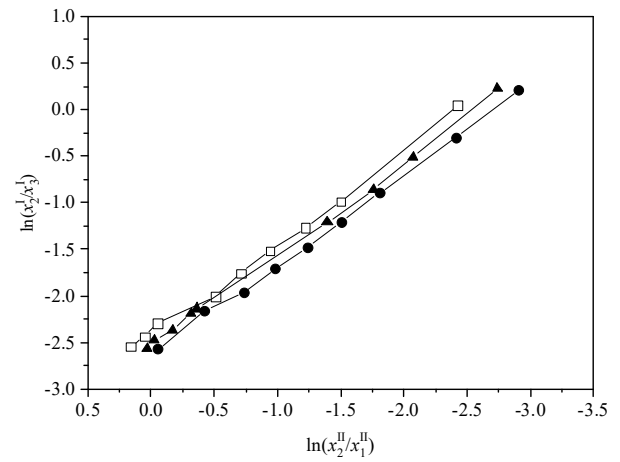

Figure 6 Hand plots for diethoxymethane at different temperatures. (口) $303.2 \mathrm{~K},(\boldsymbol{\Delta}) 313.2 \mathrm{~K}(\bullet) 323.2 \mathrm{~K}$

Table 4 The UNIQUAC structural parameters ( $\mathrm{r}$ and $\mathrm{q}$ ).

\begin{tabular}{ccc}
\hline Component & $\mathrm{r}$ & $\mathrm{q}$ \\
\hline mesityl oxide & 3.8600 & 4.3632 \\
diethoxymethane & 3.7960 & 4.3131 \\
water & 1.4000 & 0.9200
\end{tabular}

thermodynamic model NRTL and UNIQUAC equations.

$$
O F=\sum_{k=1}^{M} \sum_{j=1}^{2} \sum_{i=1}^{3}\left[\left(x_{i j k}^{e x p}-x_{i j k}^{c a l}\right)^{2}\right]
$$

Among them, $x^{\exp }$ and $x^{\text {cal }}$ represent the mole fraction of the experimental value and the calculated value, respectively. The subscripts $i, j$ and $k$ represent the composition, phase state and connection line respectively, 
and $M$ represents the total number of connection lines.

After the correlation, the binary interaction parameters of the NRTL and UNIQUAC models were obtained, as shown in Table 5; the error of the experimental value and the calculated value was compared by calculating the root mean square error (RMSD); the RMSD equation is as follows:

$$
\operatorname{RMSD}(\%)=100 \times\left\{\sum_{k=1}^{M} \sum_{j=1}^{2} \sum_{i=1}^{3} \frac{\left(x_{i j k}^{e x p}-x_{i j k}^{c a l}\right)^{2}}{6 M}\right\}^{\frac{1}{2}}
$$

Among them, the symbol in the formula has the same meaning as the formula (5). The RMSD values of the calculated and experimental values of the two models are shown in Table 5. At the same time, the calculation results of the NRTL model and the UNIQUAC model are plotted in Figure 1. It can be seen from Table 5 and Figure 1 that the experimental values have a high degree of agreement with the NRTL model and the UNIQUAC model. At the same time, the RMSD values at different temperatures are all less than $0.8 \%$, indicating that both the NRTL model and the UNIQUAC model can better correlate with the 'mesityl oxide + diethoxymethane + water' ternary system. It also shows that choosing a non-random variable $\alpha$ of 0.3 in the NRTL model is reasonable.

Using the NRTL model and the UNIQUAC model as well as the respective binary interaction parameters obtained can well predict the ternary system of 'mesityl oxide + diethoxymethane + water', which provides a basis for the design and simulation of the extraction process of diethoxymethane and water with mesityl oxide as extraction agent.

Table 5 The NRTL and UNIQUAC binary interaction parameters for the ternary system.

\begin{tabular}{|c|c|c|c|c|c|c|c|c|}
\hline \multirow[b]{2}{*}{$T / \mathrm{K}$} & \multirow[b]{2}{*}{$i-j$} & \multicolumn{3}{|c|}{ NRTL } & \multirow[b]{2}{*}{ RMSD $/ \%$} & \multicolumn{2}{|c|}{ UNIQUAC } & \multirow[b]{2}{*}{$\mathrm{RMSD} / \%$} \\
\hline & & $\underset{/ \mathrm{J} \cdot \mathrm{mol}^{-1}}{\mathrm{~g}_{\mathrm{ij}}-\mathrm{g}_{\mathrm{j}}}$ & $\begin{array}{c}\mathrm{g}_{\mathrm{ji}}-\mathrm{g}_{\mathrm{ii}} \\
/ \mathrm{J} \cdot \mathrm{mol}^{-1}\end{array}$ & $\alpha$ & & $\mathrm{u}_{\mathrm{ij}-\mathrm{u}_{\mathrm{jj}}} / \mathrm{J} \cdot \mathrm{mol}^{-1}$ & $\begin{array}{c}\mathrm{u}_{\mathrm{ji}}-\mathrm{u}_{\mathrm{ii}} \\
/ \mathrm{J} \cdot \mathrm{mol}^{-1}\end{array}$ & \\
\hline \multirow{3}{*}{303.15} & $3-1$ & 12304.73 & 3074.035 & 0.3 & \multirow{3}{*}{0.6124} & $\begin{array}{c}- \\
1292.18\end{array}$ & -2520.62 & \multirow{3}{*}{0.7948} \\
\hline & $3-2$ & 9615.765 & 6661.778 & 0.3 & & $\begin{array}{c}- \\
401.849\end{array}$ & -124.265 & \\
\hline & $1-2$ & 9071.427 & -4425.99 & 0.3 & & $\begin{array}{c}- \\
98861.5\end{array}$ & -348.835 & \\
\hline \multirow{3}{*}{313.15} & $3-1$ & 12749.47 & 3158.701 & 0.3 & \multirow{3}{*}{0.6530} & $\begin{array}{c}- \\
1351.92\end{array}$ & -2598.28 & \multirow{3}{*}{0.5863} \\
\hline & $3-2$ & 10738.12 & 5721.806 & 0.3 & & $\begin{array}{c}- \\
1029.82\end{array}$ & 485.3359 & \\
\hline & $1-2$ & 7103.242 & -3768.67 & 0.3 & & -100465 & -360.621 & \\
\hline \multirow{3}{*}{323.15} & $3-1$ & 13194.22 & 3243.368 & 0.3 & \multirow{3}{*}{0.3486} & $\begin{array}{c}- \\
1411.67\end{array}$ & -2675.95 & \multirow{3}{*}{0.2656} \\
\hline & $3-2$ & 11860.48 & 4781.833 & 0.3 & & -1657.8 & & \\
\hline & $1-2$ & 5135.057 & -3111.36 & 0.3 & & -102068 & -372.406 & \\
\hline
\end{tabular}

\section{Conclusion}

The liquid-liquid equilibrium data of 'mesityl oxide + diethoxymethane + water ternary system' at $303.15 \mathrm{~K}$, $313.15 \mathrm{~K}$ and $323.15 \mathrm{~K}$ were determined in this paper under normal pressure. It can be known from the partition coefficient and selectivity coefficient that the increase in temperature is beneficial to improve the extraction effect and selectivity of mesityl oxide. The Bachman equation and Hand equation were used to verify that the experimental data had good thermodynamic consistency. The experimental data was correlated through the NRTL and UNIQUAC models, and the binary interaction parameters between the components were obtained. At the same time, the root mean square error (RMSD) of the calculated and experimental values were both less than $0.8 \%$, indicating that the NRTL and UNIQUAC models can be better associated with experiment data. From the above analysis, it can be known that the binary interaction parameters obtained by regression using the NRTL model and the UNIQUAC model can provide a basis for the process design and simulation of the separation and recovery of diethoxymethane in water using mesityl oxide as the extractant.

\section{References}

1. N.W. Boaz, B. Venepalli. J. Org. Process Res. Dev. 5, 127-131 (2001)

2. C.H. Zhang, J.N. He, Y.L. Li, P. Li, J. Fuel. 154, 346351 (2015)

3. B. Jia, K. Xin, T.F. Yang, J.S. Yu, Y. M. Yu, Q.S. Li, J. Chem. Thermodyn. 111, 1-6 (2017)

4. H.J. Gai, C.X. Jiang, L.F. Wang, L.B. Gu, J.Q. Wang, P. CN102659204A, (2012)

5. K. Fakhri, S. Ranjbar, A. Soltanabadi, J. Mol. Liq. 299, 112225 (2020)

6. G. Busca, S. Berardinelli, C. Resini, L. Arrighi, J. Hazard. Mater. 160, 265-288 (2008)

7. J. Michałowicz, W. Duda, J. Polish J. Environ. Stud. 16, 347-362 (2007)

8. F. Wang, F. He, J. Zhao, N. Sui, L. Xu, H. Liu, J. Sep. Purif. Technol. 93, 8-14 (2012) 
9. Z. Li, K. A. Mumford, K.H. Smith, Y. Wang, G.W. Stevens, J. Sep. Sci. Technol. 49, 2913-2920 (2014)

10. S. Zhou, X. Wu, Y. Huang, X. Xie, Y. Lin, H. Fan, L. Luo, W. Zhang, J.Z. Tang, J. Ind. Crops Prod. 122, 316-328 (2018)

11. Z. Chemat-Djenni, M.A. Ferhat, V. Tomao, F.Chemat, J. J. Essent. Oil-Bearing Plants. 13, 139-147 (2010)

12. H. Dong, Y. Xian, H. Li, W. Bai, X. Zeng, J. Compr. Rev. Food Sci. Food Saf. 19, 365-404 (2020)

13. M.R. Shah, R. Anantharaj, T. Banerjee, G.D. Yadav, J. Chem. Thermodyn. 62, 142-150 (2013)

14. M. Silva, J.C. García, M. Ottens, J. Ind. Eng. Chem. Res. 57, 9210-9221 (2018)

15. L. Wang, X. Xu, Q. Li, J. Chem. Eng. 47, 34-38 (2019)
16. Y. Wang, S. Zhou, J. Tang, Y. Chen, L. Li, J. Chinese J. Chem. Eng. 26, 2573-2580 (2018)

17. J.H. Yim, K.W. Park, J.S. Lim, K.Y. Choi, J. Chem. Eng. Data. 64, 3882 - 3888 (2019)

18. C. Yang, Y. Qian, Y. Jiang, L. Zhang, J. Fluid Phase Equilib. 258, 73 - 77 (2007)

19. Q. Li, W. Fan, H. Yan, X. Huang, L. Zhang, Z. Ma, J. Fluid Phase Equilib. 505, 112353 (2020)

20. Joint Committee for Guides in Metrology, Evaluation of measurement data-Guide to the expression of uncertainty in measurement, Joint Committee for Guides in Metrology. Technical Report No. JCGM 100, 1-120 (2008)

21. Y. Pei, Q. Wang, X. Gong, F. Lei, B. Shen, J. Fluid Phase Equilib. 394, 129-139 (2015) 